\title{
Manifestation of the odd-frequency spin-triplet pairing state in diffusive ferromagnet/superconductor junctions
}

\author{
T. Yokoyama, ${ }^{1,2}$ Y. Tanaka, ${ }^{1,2}$ and A. A. Golubov ${ }^{3}$ \\ ${ }^{1}$ Department of Applied Physics, Nagoya University, Nagoya 464-8603, Japan \\ ${ }^{2}$ CREST, Japan Science and Technology Corporation (JST), Nagoya 464-8603, Japan \\ ${ }^{3}$ Faculty of Science and Technology, University of Twente, 7500 AE, Enschede, The Netherlands
}

(Received 22 October 2006; published 12 April 2007)

\begin{abstract}
Using the quasiclassical Green's function formalism, we study the influence of the odd-frequency spin-triplet superconductivity on the local density of states (LDOS) in a diffusive ferromagnet (DF) attached to a superconductor. Various possible symmetry classes in a superconductor are considered which are consistent with the Pauli's principle: even-frequency spin-singlet even-parity (ESE) state, even-frequency spin-triplet odd-parity (ETO) state, odd-frequency spin-triplet even-parity (OTE) state, and odd-frequency spin-singlet odd-parity (OSO) state. For each of these states, the pairing state in the DF is studied. Particular attention is paid to the study of spin-singlet $s$-wave and spin-triplet $p$-wave superconductors as the examples of ESE and ETO superconductors. For the spin-singlet case the magnitude of the OTE component of the pair amplitude is enhanced with the increase of the exchange field in the DF. When the OTE component is dominant at low energy, the resulting LDOS in the DF has a zero-energy peak (ZEP). On the other hand, in DF/spin-triplet $p$-wave superconductor junctions the LDOS has a ZEP in the absence of the exchange field, where only the OTE pairing state exists. With the increase of the exchange field, the ESE component of the pair amplitude induced in the DF is enhanced. Then, the resulting LDOS has a ZEP splitting. We demonstrate that the appearance of the dominant OTE component of the pair amplitude is the physical reason for the emergence of the ZEP of the LDOS.
\end{abstract}

DOI: $10.1103 /$ PhysRevB.75.134510

PACS number(s): 74.20.Rp, 74.50.+r, 74.70.Kn

\section{INTRODUCTION}

Ferromagnet/superconductor (FS) structures with conventional spin-singlet $s$-wave superconductors have been the subject of extensive work during the past decade. ${ }^{1-3}$ An exciting manifestation of the anomalous proximity effect in these structures is the existence of the so-called $\pi$ junctions in SFS Josephson junctions confirmed experimentally in Refs. 4-12. Recently, diffusive-ferromagnet/superconductor (DF/S) junctions have received much attention due to the possibility of the generation of odd-frequency pairing in these structures. ${ }^{2,13}$ In DFs, due to isotropization by the impurity scattering, only even-parity $s$-wave pairing is allowed. Besides this, the exchange field breaks the time-reversal symmetry and both spin-singlet and spin-triplet Cooper pairs can coexist. In accordance with Pauli's principle, this spintriplet state belongs to the odd-frequency spin-triplet evenparity pairing. ${ }^{2,13}$ Various aspects of this state have been addressed in recent theoretical work,,${ }^{2,14-18}$ and the first experimental observation of the long-range proximity effect due to odd-frequency pairing was reported in Refs. 19 and 20.

Odd-frequency pairing is a unique state which was first proposed by Berezinskii ${ }^{21}$ as a hypothetical state of ${ }^{3} \mathrm{He}$. The odd-frequency superconductivity was then discussed in the context of various pairing mechanisms involving strong correlations. ${ }^{22-24}$ However, the proximity effect in the presence of an odd-frequency superconducting state has not been studied until very recently.

A general theory of the proximity effect in junctions composed of a diffusive normal (DN) metal and unconventional superconductor in the framework of the quasiclassical
Green's function formalism was recently presented by two of the present authors. ${ }^{25}$ Various possible symmetry classes in a superconductor were considered in Ref. 25 which are consistent with Pauli's principle: the even-frequency spin-singlet even-parity (ESE) state, even-frequency spin-triplet oddparity (ETO) state, odd-frequency spin-triplet even-parity (OTE) state, and odd-frequency spin-singlet odd-parity (OSO) state. For each of the above four cases, the symmetry and spectral properties of the induced pair amplitude in the DN metal were determined. It was shown that the pair amplitude in a DN metal belongs, respectively, to ESE, OTE, OTE, and ESE pairing states. It is remarkable that the OTE state is realized without assuming magnetic ordering in DN/ ETO superconductor junctions, where the midgap Andreev resonant state $^{26}$ formed at the interface penetrates into the DN metal and the resulting local density of states (LDOS) has a zero-energy peak (ZEP). ${ }^{27}$

On the other hand, the existence of the ZEP in the LDOS in the DF/ESE $s$-wave superconductor junctions has been established. ${ }^{5,28-32}$ Although the conditions of the formation of the ZEP in DF regions were formulated by the present authors, ${ }^{33}$ the possible relation between the ZEP and the formation of OTE pairing in the DF has not yet been clarified. The present paper addresses this issue. We also study the proximity effect in DF/ETO $p$-wave superconductor junctions. It was shown in the previous paper ${ }^{25}$ that only the OTE pairing state is generated without exchange field $h$. It is an interesting question how this unusual proximity effect is influenced by the exchange field.

The organization of the present paper is as follows. In Sec. II, we formulate the proximity effect model in DF/S junctions within the theory applicable to unconventional su- 
perconductor junctions where the midgap Andreev resonant states are naturally taken into account in the boundary condition for the quasiclassical Green's function. ${ }^{27}$ We discuss the general properties of the proximity effect by choosing ESE, ETO, OTE, and OSO superconductor junctions. It is clarified that the OTE, ESE, ESE, and OTE states are, respectively, generated in the DF in the presence of exchange field $h$. In Sec. III we calculate the pair amplitude in the DF for spin-singlet $s$-wave and spin-triplet $p$-wave superconductor junctions as an example of ESE and ETO superconductor junctions. For $s$-wave junctions, it is revealed that generation of the OTE pairing state by the exchange field $h$ causes an enhancement of the zero-energy LDOS in the DF. On the other hand, for $p$-wave superconductor junctions, generation of the ESE pairing state by $h$ results in a splitting of the ZEP of the LDOS. We clarify the relation between the ZEP in the LDOS and the generation of the OTE state in the DF. A summary of the results is given in Sec. IV.

\section{FORMULATION}

Let us start with the formulation of the general symmetry properties of the quasiclassical Green's functions in the considered system following the discussion in Ref. 25. The elements of retarded and advanced Nambu matrices $\hat{g}^{R, A}$,

$$
\hat{g}^{R, A}=\left(\begin{array}{ll}
g^{R, A} & f^{R, A} \\
\tilde{f}^{R, A} & \tilde{g}^{R, A}
\end{array}\right),
$$

are composed of normal $g_{\alpha, \beta}^{R}(\boldsymbol{r}, \varepsilon, \boldsymbol{p})$ and anomalous $f_{\alpha, \beta}^{R}(\boldsymbol{r}, \varepsilon, \boldsymbol{p})$ components with spin indices $\alpha$ and $\beta$. Here $\boldsymbol{p}$ $=\boldsymbol{p}_{F} /\left|\boldsymbol{p}_{F}\right|, \boldsymbol{p}_{F}$ is the Fermi momentum, and $\boldsymbol{r}$ and $\varepsilon$ denote the coordinate and energy of a quasiparticle measured from the Fermi level, respectively. The function $f^{R}$ and the conjugated function $\widetilde{f}^{R}$ satisfy the following relation: $:^{34,35}$

$$
\tilde{f}_{\alpha, \beta}^{R}(\boldsymbol{r}, \varepsilon, \boldsymbol{p})=-\left[f_{\alpha, \beta}^{R}(\boldsymbol{r},-\varepsilon,-\boldsymbol{p})\right]^{*} .
$$

Pauli's principle is formulated in terms of the retarded and advanced Green's functions in the following way: ${ }^{34}$

$$
f_{\alpha, \beta}^{A}(\boldsymbol{r}, \varepsilon, \boldsymbol{p})=-f_{\beta, \alpha}^{R}(\boldsymbol{r},-\varepsilon,-\boldsymbol{p}) .
$$

By combining the above two equations, we obtain $\tilde{f}_{\beta, \alpha}^{R}(\boldsymbol{r}, \varepsilon, \boldsymbol{p})=\left[f_{\alpha, \beta}^{A}(\boldsymbol{r}, \varepsilon, \boldsymbol{p})\right]^{*}$. Further, the definitions of the even-frequency and odd-frequency pairings are $f_{\alpha, \beta}^{A}(\boldsymbol{r}, \varepsilon, \boldsymbol{p})$ $=f_{\alpha, \beta}^{R}(\boldsymbol{r},-\varepsilon, \boldsymbol{p})$ and $f_{\alpha, \beta}^{A}(\boldsymbol{r}, \varepsilon, \boldsymbol{p})=-f_{\alpha, \beta}^{R}(\boldsymbol{r},-\varepsilon, \boldsymbol{p})$, respectively. Finally we get

$$
\widetilde{f}_{\beta, \alpha}^{R}(\boldsymbol{r}, \varepsilon, \boldsymbol{p})=\left[f_{\alpha, \beta}^{R}(\boldsymbol{r},-\varepsilon, \boldsymbol{p})\right]^{*}
$$

for the even-frequency pairing and

$$
\tilde{f}_{\beta, \alpha}^{R}(\boldsymbol{r}, \varepsilon, \boldsymbol{p})=-\left[f_{\alpha, \beta}^{R}(\boldsymbol{r},-\varepsilon, \boldsymbol{p})\right]^{*}
$$

for the odd-frequency pairing. In the following, we consider homogeneous ferromagnet/superconductor junctions with exchange field $h$ in a ferromagnet and focus on the Cooper pairs with $S_{z}=0$. In this case, it is possible to remove the external phase of the pair potential in the superconductor. We will concentrate on the retarded part of the Green's function.
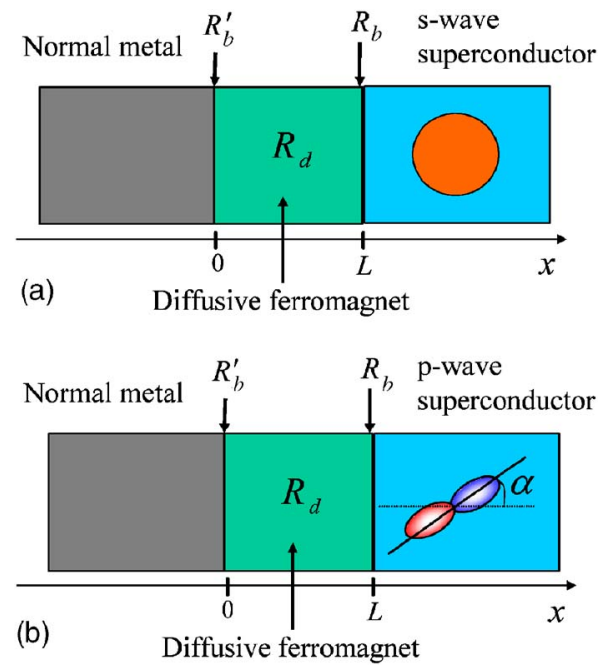

FIG. 1. (Color online) Schematic illustration of DF/S junctions where the DF is connected to normal reservoirs. (a) Conventional spin-singlet $s$-wave superconductor and (b) spin-triplet $p$-wave superconductor junctions.

We consider a junction consisting of a normal $(\mathrm{N})$ and a superconducting reservoir connected by a quasi-onedimensional DF with a length $L$ much larger than the mean free path as shown in Fig. 1.

The interface between the DF and the superconductor (S) at $x=L$ has a resistance $R_{b}$ and the N/DF interface at $x=0$ has a resistance $R_{b}^{\prime}$. The Green's function in the superconductor can be parametrized as $g_{ \pm}(\varepsilon) \hat{\tau}_{3}+f_{ \pm}(\varepsilon) \hat{\tau}_{2}$ using Pauli's matrices, where the subscript $+(-)$ denotes the right- (left-) going quasiparticles. $g_{ \pm}(\varepsilon)$ and $f_{ \pm}(\varepsilon)$ are given by $g_{+}(\varepsilon)$ $\equiv g_{\uparrow, \uparrow}^{R}(\boldsymbol{r}, \varepsilon, \boldsymbol{p})=g_{\downarrow, \downarrow}^{R}(\boldsymbol{r}, \boldsymbol{\varepsilon}, \boldsymbol{p}), g_{-}(\varepsilon) \equiv g_{\uparrow, \uparrow}^{R}(\boldsymbol{r}, \varepsilon, \overline{\boldsymbol{p}})=g_{\downarrow, \downarrow}^{R}(\boldsymbol{r}, \varepsilon, \overline{\boldsymbol{p}})$, $f_{+}(\varepsilon) \equiv f_{\uparrow, \downarrow}^{R}(\boldsymbol{r}, \varepsilon, \boldsymbol{p})$, and $f_{-}(\varepsilon) \equiv f_{\uparrow, \downarrow}^{R}(\boldsymbol{r}, \varepsilon, \overline{\boldsymbol{p}})$, respectively, with $\overline{\boldsymbol{p}}=\overline{\boldsymbol{p}}_{F} /\left|\boldsymbol{p}_{F}\right|$ and $\overline{\boldsymbol{p}}_{F}=\left(-p_{F x}, p_{F y}\right)$. Using relations (4) and (5), we obtain that $f_{ \pm}(\varepsilon)=\left[f_{ \pm}(-\varepsilon)\right]^{*}$ for the even-frequency pairing and $f_{ \pm}(\varepsilon)=-\left[f_{ \pm}(-\varepsilon)\right]^{*}$ for the odd-frequency pairing, respectively, while $g_{ \pm}(\varepsilon)=\left[g_{ \pm}(-\varepsilon)\right]^{*}$ in both cases.

In the DF region, only the $s$-wave even-parity pairing state is allowed due to isotropization by impurity scattering. The resulting Green's function with majority and minority spin in the DF can be parametrized by $\cos \theta \hat{\tau}_{3}+\sin \theta \hat{\tau}_{2}$ and $\cos \bar{\theta} \hat{\tau}_{3}+\sin \bar{\theta} \hat{\tau}_{2}$ in a junction with an even-parity superconductor, respectively. On the other hand, for an odd-parity superconductor, the corresponding quantities for majority spin and minority spin are expressed by $\cos \theta \hat{\tau}_{3}+\sin \theta \hat{\tau}_{1}$ and $\cos \bar{\theta} \hat{\tau}_{3}+\sin \bar{\theta} \hat{\tau}_{1}$, respectively.

The function $\theta$ satisfies the Usadel equation ${ }^{36}$

$$
D \frac{\partial^{2} \theta}{\partial x^{2}}+2 i(\varepsilon+h) \sin \theta=0
$$

with the boundary conditions at the DF/S interface, ${ }^{27,37}$

$$
\left.\frac{L}{R_{d}}\left(\frac{\partial \theta}{\partial x}\right)\right|_{x=L}=\frac{\left\langle F_{1}\right\rangle}{R_{b}}
$$




$$
F_{1}=\frac{2 T_{1}\left(f_{S} \cos \theta_{L}-g_{S} \sin \theta_{L}\right)}{2-T_{1}+T_{1}\left(\cos \theta_{L} g_{S}+\sin \theta_{L} f_{S}\right)},
$$

and at the N/DF interface,

$$
\left.\frac{L}{R_{d}}\left(\frac{\partial \theta}{\partial x}\right)\right|_{x=0}=-\frac{\left\langle F_{2}\right\rangle}{R_{b}^{\prime}}, \quad F_{2}=\frac{2 T_{2} \sin \theta_{0}}{2-T_{2}+T_{2} \cos \theta_{0}},
$$

respectively, with $\theta_{L}=\left.\theta\right|_{x=L}$ and $\theta_{0}=\left.\theta\right|_{x=0}$. Here, $R_{d}$ and $D$ are the resistance and the diffusion constant in the DF, respectively. The function $g_{S}$ is given by $g_{S}=\left(g_{+}+g_{-}\right) /(1$ $\left.+g_{+} g_{-}+f_{+} f_{-}\right)$and $f_{S}=\left(f_{+}+f_{-}\right) /\left(1+g_{+} g_{-}+f_{+} f_{-}\right)$for the evenparity pairing and $f_{S}=i\left(f_{+} g_{-}-f_{-} g_{+}\right) /\left(1+g_{+} g_{-}+f_{+} f_{-}\right)$for the odd-parity pairing, respectively, with $g_{ \pm}=\varepsilon / \sqrt{\varepsilon^{2}-\Delta_{ \pm}^{2}}, f_{ \pm}$ $=\Delta_{ \pm} / \sqrt{\Delta_{ \pm}^{2}-\varepsilon^{2}}$, and $\Delta_{ \pm}=\Delta \Psi\left(\phi_{ \pm}\right)$, where $\Psi\left(\phi_{ \pm}\right)$is the form factor with $\phi_{+}=\phi$ and $\phi_{-}=\pi-\phi$. The brackets $\langle\cdots\rangle$ denote averaging over the injection angle $\phi$ :

$$
\begin{gathered}
\left\langle F_{1(2)}(\phi)\right\rangle=\int_{-\pi / 2}^{\pi / 2} d \phi \cos \phi F_{1(2)}(\phi) / \int_{-\pi / 2}^{\pi / 2} d \phi T_{1(2)} \cos \phi \\
T_{1}=\frac{4 \cos ^{2} \phi}{Z^{2}+4 \cos ^{2} \phi}, \quad T_{2}=\frac{4 \cos ^{2} \phi}{Z^{\prime 2}+4 \cos ^{2} \phi}
\end{gathered}
$$

where $T_{1,2}$ are the transmission probabilities and $Z$ and $Z^{\prime}$ are the barrier parameters for two interfaces.

The resistance at the interface $R_{b}^{(\prime)}$ is given by

$$
R_{b}^{(\prime)}=\frac{2 R_{0}^{(\prime)}}{\int_{-\pi / 2}^{\pi / 2} d \phi T_{1(2)}(\phi) \cos \phi} .
$$

Here, $R_{b}^{(\prime)}$ denotes $R_{b}$ or $R_{b}^{\prime}$ and $R_{0}^{(\prime)}$ is Sharvin resistance, which in the three-dimensional case is given by $R_{0}^{(\prime)}$ $=4 \pi^{2} /\left(e^{2} k_{F}^{2} S_{c}^{(\prime)}\right)$, where $k_{F}$ is the Fermi wave vector and $S_{c}^{(\prime)}$ is the constriction area.

Next, we focus on the Green's function of minority spin. The function $\bar{\theta}$ satisfies the following equation: ${ }^{36}$

$$
D \frac{\partial^{2} \bar{\theta}}{\partial x^{2}}+2 i(\varepsilon-h) \sin \bar{\theta}=0
$$

with the boundary condition at the DF/S interface, ${ }^{27,37}$

$$
\left.\frac{L}{R_{d}}\left(\frac{\partial \bar{\theta}}{\partial x}\right)\right|_{x=L}=\frac{\left\langle\bar{F}_{1}\right\rangle}{R_{b}}
$$

Here, $\bar{F}_{1}$ is given by

$$
\bar{F}_{1}=\frac{2 T_{1}\left(f_{S} \cos \bar{\theta}_{L}-g_{S} \sin \bar{\theta}_{L}\right)}{2-T_{1}+T_{1}\left(\cos \bar{\theta}_{L} g_{S}+\sin \bar{\theta}_{L} f_{S}\right)}
$$

for a spin-triplet superconductor and

$$
\bar{F}_{1}=\frac{2 T_{1}\left(-f_{S} \cos \bar{\theta}_{L}-g_{S} \sin \bar{\theta}_{L}\right)}{2-T_{1}+T_{1}\left(\cos \bar{\theta}_{L} g_{S}-\sin \bar{\theta}_{L} f_{S}\right)}
$$

for a spin-singlet superconductor, respectively. At the N/DF interface, the boundary condition reads

$$
\left.\frac{L}{R_{d}}\left(\frac{\partial \bar{\theta}}{\partial x}\right)\right|_{x=0}=-\frac{\left\langle\bar{F}_{2}\right\rangle}{R_{b}^{\prime}}, \quad \bar{F}_{2}=\frac{2 T_{2} \sin \bar{\theta}_{0}}{2-T_{2}+T_{2} \cos \bar{\theta}_{0}} .
$$

Here $\bar{\theta}_{L}=\left.\bar{\theta}\right|_{x=L}$ and $\bar{\theta}_{0}=\left.\bar{\theta}\right|_{x=0}$.

Equations (12), (13), and (16) can be transformed into

$$
\begin{gathered}
D \frac{\partial^{2} \bar{\theta}^{*}(-\varepsilon)}{\partial x^{2}}+2 i(\varepsilon+h) \sin \bar{\theta}^{*}(-\varepsilon)=0, \\
\left.\frac{L}{R_{d}}\left(\frac{\partial \bar{\theta}^{*}(-\varepsilon)}{\partial x}\right)\right|_{x=L}=\frac{\left\langle\bar{F}_{1}^{*}(-\varepsilon)\right\rangle}{R_{b}}, \\
\left.\frac{L}{R_{d}}\left(\frac{\partial \bar{\theta}^{*}(-\varepsilon)}{\partial x}\right)\right|_{x=0}=-\frac{\left\langle\bar{F}_{2}^{*}(-\varepsilon)\right\rangle}{R_{b}^{\prime}} .
\end{gathered}
$$

The pair amplitude is defined as

$$
f_{3}(\varepsilon)=(\sin \theta-\sin \bar{\theta}) / 2
$$

in the spin-singlet case and as

$$
f_{0}(\varepsilon)=(\sin \theta+\sin \bar{\theta}) / 2
$$

in the spin-triplet case.

Since only an even-parity $s$-wave pairing can exist in the DF due to the impurity scattering, $f_{3}$ and $f_{0}$ belong to the ESE and OTE states, respectively.

In the following, we will consider four possible symmetry classes of superconductivity in the junction, consistent with Pauli's principle: ESE, ETO, OTE, and OSO pairing states.

(i) Junction with ESE superconductor. In this case, $f_{ \pm}(\varepsilon)$ $=f_{ \pm}^{*}(-\varepsilon)$ and $g_{ \pm}(\varepsilon)=g_{ \pm}^{*}(-\varepsilon)$ are satisfied. Then, $f_{S}(-\varepsilon)$ $=f_{S}^{*}(\varepsilon)=f_{S}^{*}$ and $g_{S}(-\varepsilon)=g_{S}^{*}(\varepsilon)=g_{S}^{*}$ and we obtain for $\bar{F}_{1}^{*}(-\varepsilon)$

$$
\bar{F}_{1}^{*}(-\varepsilon)=\frac{2 T_{1}\left[-f_{S} \cos \bar{\theta}_{L}^{*}(-\varepsilon)-g_{S} \sin \bar{\theta}_{L}^{*}(-\varepsilon)\right]}{2-T_{1}+T_{1}\left[\cos \bar{\theta}_{L}^{*}(-\varepsilon) g_{S}-\sin \bar{\theta}_{L}^{*}(-\varepsilon) f_{S}\right]} .
$$

It follows from a comparison of Eqs. (6)-(9) with Eqs. (17)-(19) that these equations are consistent with each other only when $\sin \bar{\theta}^{*}(-\varepsilon)=-\sin \theta(\varepsilon)$ and $\cos \bar{\theta}^{*}(-\varepsilon)=\cos \theta(\varepsilon)$. After a simple calculation, we can show $f_{3}(\varepsilon)=f_{3}^{*}(-\varepsilon)$ and $f_{0}(\varepsilon)=-f_{0}^{*}(-\varepsilon)$. This relation is consistent with the fact ${ }^{25}$ that $f_{3}$ and $f_{0}$ are the even-frequency and odd-frequency pairing states, respectively. When $h=0$, since $\sin \theta(\varepsilon)=-\sin \bar{\theta}(\varepsilon)$ is satisfied, the resulting $f_{0}$ is vanishing and only the ESE state exists. For $h \neq 0, f_{0}$ becomes nonzero and the OTE state is generated in the DF.

(ii) Junction with ETO superconductor. Now we have $f_{ \pm}(\varepsilon)=f_{ \pm}^{*}(-\varepsilon)$ and $g_{ \pm}(\varepsilon)=g_{ \pm}^{*}(-\varepsilon)$. Then, $f_{S}(-\varepsilon)=-f_{S}^{*}(\varepsilon)=-f_{S}^{*}$ and $g_{S}(-\varepsilon)=g_{S}^{*}(\varepsilon)=g_{S}^{*}$. As a result, $\bar{F}_{1}^{*}(-\varepsilon)$ is given by 

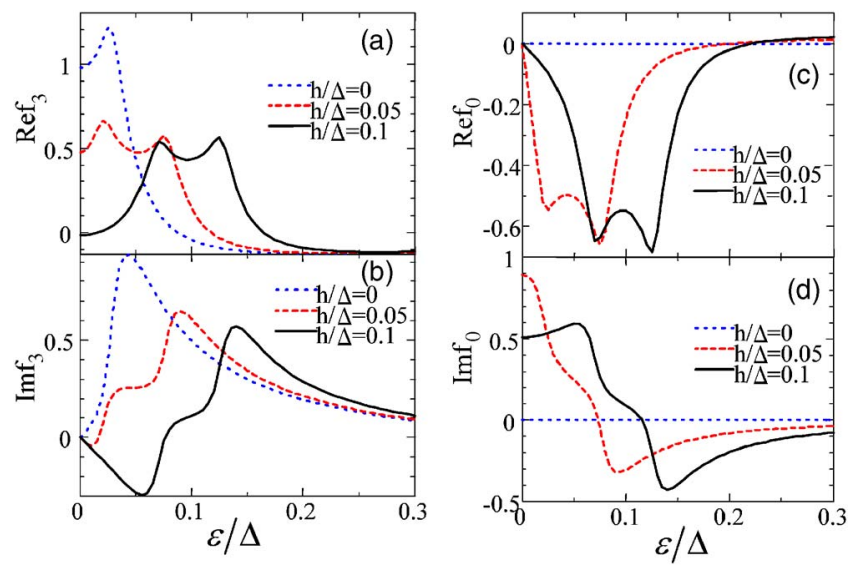

FIG. 2. (Color online) Real (a) and imaginary (b) parts of $f_{3}$ and real (c) and imaginary (d) parts of $f_{0}$ in spin-singlet $s$-wave superconductor junctions. We choose $R_{d} / R_{b}=1$.

$$
\bar{F}_{1}^{*}(-\varepsilon)=-\frac{2 T_{1}\left[f_{S} \cos \bar{\theta}_{L}^{*}(-\varepsilon)+g_{S} \sin \bar{\theta}_{L}^{*}(-\varepsilon)\right]}{2-T_{1}+T_{1}\left[\cos \bar{\theta}_{L}^{*}(-\varepsilon) g_{S}-\sin \bar{\theta}_{L}^{*}(-\varepsilon) f_{S}\right]} .
$$

Equations (6)-(9) and (17)-(19) are consistent if $\sin \theta^{*}(-\varepsilon)$ $=-\sin \bar{\theta}(\varepsilon)$ and $\cos \theta^{*}(-\varepsilon)=\cos \bar{\theta}(\varepsilon)$. As in the case of ESE pairing, we can show $f_{3}(\varepsilon)=f_{3}^{*}(-\varepsilon)$ and $f_{0}(\varepsilon)=-f_{0}^{*}(-\varepsilon)$. For $h=0$, the OTE state is generated in the DF as shown in our recent paper. ${ }^{25}$ The ESE state is generated by $h$, in contrast to the case of DF/ESE superconductor junctions.

(iii) Junction with OTE superconductor. In this case $f_{ \pm}(\varepsilon)=-f_{ \pm}^{*}(-\varepsilon)$ and $g_{ \pm}(\varepsilon)=g_{ \pm}^{*}(-\varepsilon)$. Then $f_{S}(-\varepsilon)=-f_{S}^{*}(\varepsilon)$ and $g_{S}(-\varepsilon)=g_{S}^{*}(\varepsilon)$ and one can show that $\bar{F}_{1}^{*}(-\varepsilon)$ has the same form as in the case of ESE and ETO superconductor junctions. Then, we obtain $\sin \bar{\theta}^{*}(-\varepsilon)=-\sin \theta(\varepsilon)$ and $\cos \bar{\theta}^{*}(-\varepsilon)$ $=\cos \theta(\varepsilon)$. Also $f_{3}(\varepsilon)=f_{3}^{*}(-\varepsilon)$ and $f_{0}(\varepsilon)=-f_{0}^{*}(-\varepsilon)$ are satisfied. For $h=0$, only the OTE pairing state is generated in the DF. Similar to the case of ETO junctions, ESE pairing is induced in the presence of $h$.

(iv) Junction with OSO superconductor. We have $f_{ \pm}(\varepsilon)$ $=-f_{ \pm}^{*}(-\varepsilon), g_{ \pm}(\varepsilon)=g_{ \pm}^{*}(-\varepsilon), f_{S}(-\varepsilon)=f_{S}^{*}(\varepsilon)$, and $g_{S}(-\varepsilon)=g_{S}^{*}(\varepsilon)$. One can show that $\bar{F}_{1}^{*}(-\varepsilon)$ takes the same form as in the cases of ESE, ETO, and OTE superconductor junctions. Then, we obtain $\sin \bar{\theta}^{*}(-\varepsilon)=-\sin \theta(\varepsilon)$ and $\cos \bar{\theta}^{*}(-\varepsilon)$ $=\cos \theta(\varepsilon)$. Also $f_{3}(\varepsilon)=f_{3}^{*}(-\varepsilon)$ and $f_{0}(\varepsilon)=-f_{0}^{*}(-\varepsilon)$ are satisfied. For $h=0$, only the ESE pairing state is generated in the DF. Similar to the case of ETO junctions, OTE pairing is induced in the presence of $h$.

We can now summarize the above results in the table below. As seen from the above discussion, $\sin \bar{\theta}^{*}(-\varepsilon)$ $=-\sin \theta(\varepsilon), \cos \bar{\theta}^{*}(-\varepsilon)=\cos \theta(\varepsilon), f_{3}(\varepsilon)=f_{3}^{*}(-\varepsilon)$, and $f_{0}(\varepsilon)=$ $-f_{0}^{*}(-\varepsilon)$ are satisfied for all cases. The real part of $f_{3}$ is an even function of $\varepsilon$ while the imaginary part of it is an odd function of $\varepsilon$ consistent with even-frequency pairing. On the other hand, the real part of $f_{0}$ is an odd function of $\varepsilon$ while its imaginary part is an even function of $\varepsilon$ consistent with oddfrequency pairing:
Symmetry of

Symmetry of the pairing in

the pairing in

DF without

superconductors

exchange field

Symmetry of

the pairing in

DF

(i)

Even-
frequency
sin-singlet
even-parity
(ESE)

(ii)

Even-

ESE

$\mathrm{ESE}+\mathrm{OTE}$

frequency

spin-triplet

odd-parity

(ETO)

(iii)

Odd-

frequency

spin-triplet

even-parity

(OTE)

Odd-

frequency

spin-singlet

odd-parity

(OSO)

Within this formulation, the LDOS in the DF layer is given by

$$
N / N_{0}=\frac{1}{2}(\operatorname{Re} \cos \theta+\operatorname{Re} \cos \bar{\theta}),
$$

where $N_{0}$ denotes the LDOS in the normal state. Below we will calculate $f_{3}$ and $f_{0}$ and the LDOS at zero temperature. For this purpose, we will use the parameter set $Z=3, Z^{\prime}=3$, $E_{T h} \equiv D / L^{2}=0.1 \Delta$, and $R_{d} / R_{b}^{\prime}=0.1$, which represents a typical DF/S junction. Our qualitative conclusions are not sensitive to the parameter choice.

\section{RESULTS}

In the following, we will study two typical cases. As an example of an ESE superconductor, conventional spinsinglet $s$-wave pairing will be considered. We will clarify the generation of OTE pairing in the DF by the exchange field $h$ consistent with preexisting results. ${ }^{2,13}$ We will also study the spin-triplet $p$-wave superconductor as a typical example of an ETO superconductor. In this case, the ESE pairing state is induced by $h$. It should be remarked again that $f_{3}$ and $f_{0}$ denote the ESE and OTE pairing amplitudes, respectively.

\section{A. Spin-singlet $s$-wave superconductor junctions}

Let us first study DF/spin-singlet $s$-wave superconductor junctions where we choose $R_{d} / R_{b}=1$ and the form factor $\Psi_{ \pm}$ is given by $\Psi_{ \pm}=1$. The real and imaginary parts of $f_{3}$ and $f_{0}$ at $x=0$ for various $h / \Delta$ are shown in Fig. 2. Without an exchange field-i.e., $h=0$ - only the $f_{3}$ is nonzero, consistent with conventional theory of the proximity effect. ${ }^{37-39}$ By introducing the exchange field $h$, the magnitude of $f_{3}$ is sup- 


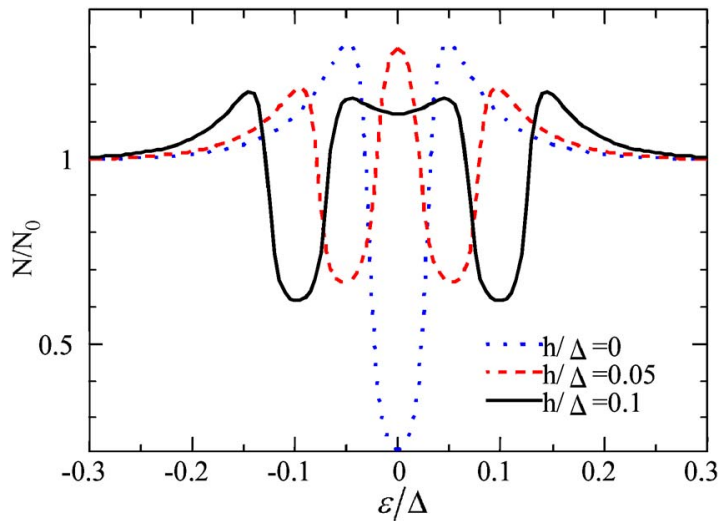

FIG. 3. (Color online) Normalized LDOS as a function of $\varepsilon$ for $R_{d} / R_{b}=1$ with various $h / \Delta$ in spin-singlet $s$-wave superconductor junctions.

pressed for small $\varepsilon$ while it is enhanced for large $\varepsilon$ as shown in Figs. 2(a) and 2(b). On the other hand, the imaginary part of $f_{0}$ is enhanced for a small magnitude of $\varepsilon$. The corresponding LDOS at the N/DF interface normalized by its value in the normal state is plotted as a function of $\varepsilon$ in Fig. 3. The LDOS has a minigap at $h=0 .{ }^{38,39}$ As shown in Fig. 3, the LDOS is influenced crucially by $h$. A peak appears at zero energy with $h / \Delta=0.05$. In this case $\operatorname{Im} f_{0}$ has a large value at zero energy as shown in Fig. 2(d). Thus the large magnitude of $\operatorname{Im} f_{0}$ at $\varepsilon=0$ is responsible for the peak of the LDOS.

It was shown in our previous work ${ }^{33}$ that the condition for the formation of a ZEP in the LDOS is given by $E_{T h}$ $\sim 2 h R_{b} / R_{d}$. This condition is consistent with the results shown in Fig. 3. As shown in Fig. 2, when this condition is satisfied, $\operatorname{Im} f_{0}$ has a large value at the zero energy. Thus it corresponds to the generation of the odd-frequency pairing amplitude $f_{0}$ at low energy. The spatial dependences of the pair amplitudes $f_{3}$ and $f_{0}$ at $\varepsilon=0$ are shown in Fig. 4. The

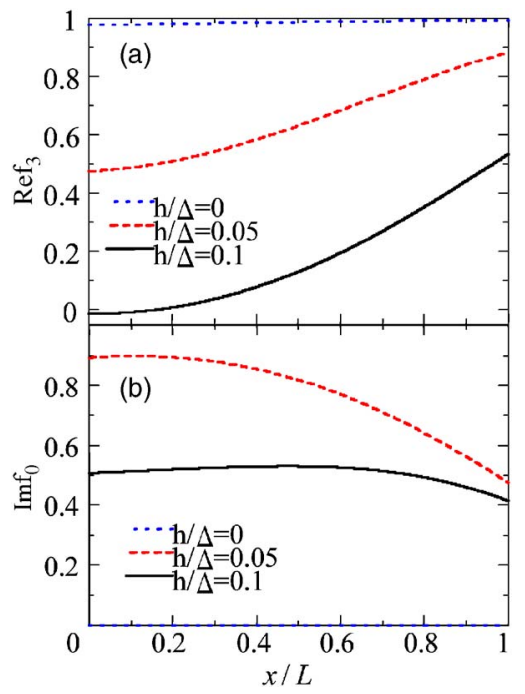

FIG. 4. (Color online) Spatial dependence of the pair amplitudes $f_{3}$ and $f_{0}$ in the DF for $\varepsilon=0$ in spin-singlet $s$-wave superconductor junctions. For $\varepsilon=0, \operatorname{Im} f_{3}=0$ and $\operatorname{Re} f_{0}=0$ are satisfied.

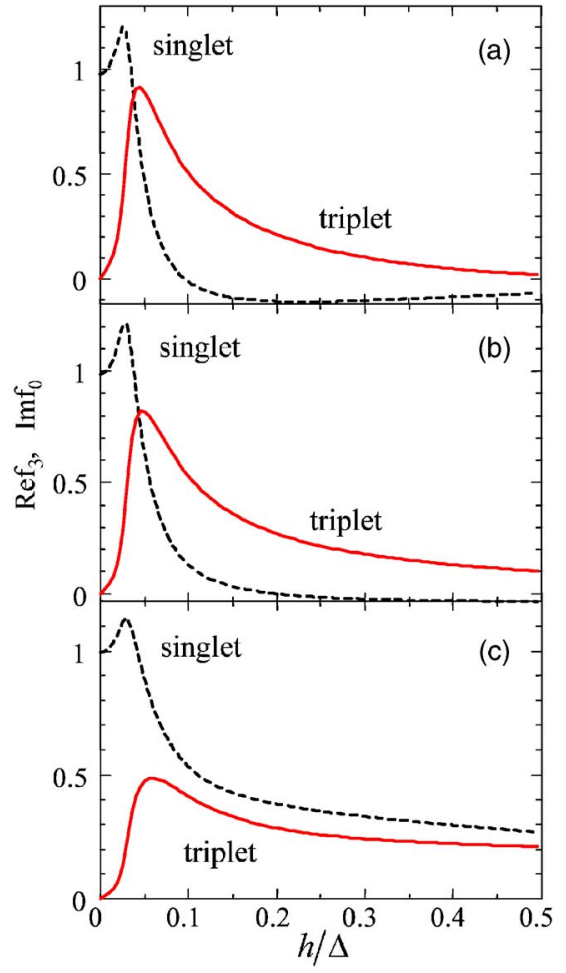

FIG. 5. (Color online) The pair amplitudes $f_{3}$ and $f_{0}$ as a function of $h$ in the DF for $\varepsilon=0$ in spin-singlet $s$-wave superconductor junctions. (a) $x=0$, (b) $x=L / 2$, and (c) $x=L$.

amplitude of $f_{3}$ is dominant near the DF/S interface while the magnitude of $f_{0}$ is enhanced at the N/DF interface.

Let us study the crossover between singlet and triplet pairing states. We show $f_{3}$ and $f_{0}$ as a function of $h$ for $\varepsilon=0$ at (a) $x=0$, (b) $x=L / 2$, and (c) $x=L$ in Fig. 5. $f_{0}$ increases from zero with $h$. At a certain value of $h, f_{0}$ has a maximum. If the value of $h$ is larger than this value, the triplet component becomes dominant as shown in Figs. 5(a) and 5(b). The value of $h$ at the crossover regime is given by the minigap in DN/S junctions. Let us discuss this regime in more detail. As shown in Sec. II, $\sin \bar{\theta}(\varepsilon)=-\sin \theta^{*}(-\varepsilon)$ and $\cos \bar{\theta}(\varepsilon)$ $=\cos \theta^{*}(-\varepsilon)$ are satisfied for any case. Then the ESE and OTE pair wave functions in the DF are given by

$$
\begin{aligned}
& f_{3}(\varepsilon)=\left[\sin \theta(\varepsilon)+\sin \theta^{*}(-\varepsilon)\right] / 2, \\
& f_{0}(\varepsilon)=\left[\sin \theta(\varepsilon)-\sin \theta^{*}(-\varepsilon)\right] / 2 .
\end{aligned}
$$

At $\varepsilon=0$, we denote $\theta(0)=\operatorname{Re} \theta(0)+i \operatorname{Im} \theta(0)$, where $\operatorname{Re} \theta(0)$ and $\operatorname{Im} \theta(0)$ are the real and imaginary parts of $\theta(0)$. Then $f_{3}(0)$ and $f_{0}(0)$ are given by $\cosh [\operatorname{Im} \theta(0)] \sin [\operatorname{Re} \theta(0)]$ and $i \sinh [\operatorname{Im} \theta(0)] \cos [\operatorname{Re} \theta(0)]$. Thus the following equation is satisfied:

$$
\frac{f_{3}(0)}{f_{0}(0)}=\frac{\tan \operatorname{Re} \theta(0)}{i \tanh \operatorname{Im} \theta(0)} .
$$

It is easy to show that $|\operatorname{Re} \theta(0)|<|\operatorname{Im} \theta(0)|$ is satisfied when the crossover occurs-i.e., $\tan \operatorname{Re} \theta(0)=\tanh \operatorname{Im} \theta(0)$. As shown in our previous work, ${ }^{33}$ this inequality is satisfied 

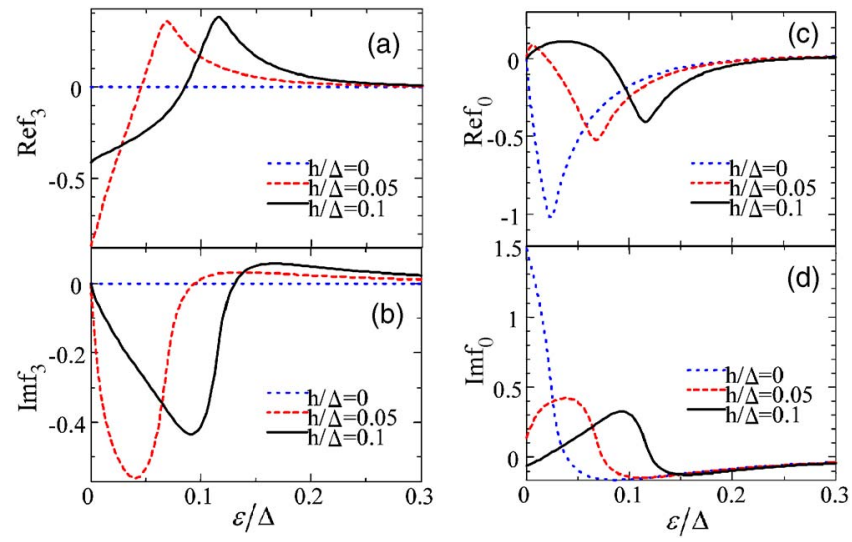

FIG. 6. (Color online) Pair amplitudes for DF/spin-triplet $p$-wave superconductor junctions. Real (a) and imaginary (b) parts of $f_{3}$. Real (c) and imaginary (d) parts of $f_{0}$. Here we choose $R_{d} / R_{b}=0.1$.

when the exchange field is of the order of the minigap energy in DN/S junctions-i.e., $h \sim\left(R_{d} / R_{b}\right)\left(E_{T h} / 2\right)$. Therefore the crossover occurs around this value of the exchange field.

\section{B. Spin-triplet $p$-wave superconductor junctions}

Next we focus on the $\mathrm{DF} /$ spin-triplet $p$-wave superconductor junctions, where we choose $R_{d} / R_{b}=0.1$ and the form factor $\Psi_{ \pm}$is given by $\Psi_{ \pm}= \pm \cos \phi$ corresponding to the case of $\alpha=0$ (see Fig. 1). In order to make numerical calculations stable, we introduce a small imaginary number in the quasiparticle energy: $\varepsilon \rightarrow \varepsilon+i \gamma$, with $\gamma=0.01 \Delta$. The real and imaginary parts of $f_{3}$ and $f_{0}$ at $x=0$ are plotted in Fig. 6 for various $h / \Delta$. Similar to the case of DN/s-wave superconductor junctions, the imaginary part of $f_{3}$ and the real part of $f_{0}$ vanish at $\varepsilon=0$. For $h=0, f_{3}=0$ and only $f_{0}$ is nonzero as shown in Fig. 6. The feature of this unusual proximity effect $^{27}$ was already discussed in our previous paper, ${ }^{25}$ where the OTE pairing state is generated in the DN of DN/ETO superconductor junctions. In this case, the LDOS has a ZEP and the odd-frequency component $f_{0}$ becomes a purely imaginary number at $\varepsilon=0$. With increasing $h$, the amplitude of $f_{3}$ is enhanced as shown in Figs. 6(a) and 6(b), in contrast to the case of DN/spin-singlet $s$-wave superconductor junctions. At the same time, the magnitude of $f_{0}$ near zero energy is suppressed. Then the features of the proximity effect in the $\mathrm{DF}$ are the same as in conventional superconductor junctions. The corresponding LDOS normalized by its value in the normal state is plotted as a function of $\varepsilon$ in Fig. 7. With the increase of $h$, the magnitude of the LDOS at $\varepsilon=0$ is suppressed and the LDOS peak is split. The magnitude of the splitting increases with the increase of $h$. Note that the peak positions in $\operatorname{Im} f_{0}$ and LDOS coincide with each other. The spatial dependences of the real part of $f_{3}$ and the imaginary part of $f_{0}$ at $\varepsilon=0$ are shown in Fig. 8. For $h=0, f_{3}$ is absent and the magnitude of the imaginary part of $f_{0}$ reaches its maximum at the DF/S interface. With the increase of $h$, the amplitude of $f_{0}$ is drastically reduced. The spatial dependence of $f_{3}$ is rather weak, and its amplitude is most strongly enhanced for $h=0.05 \Delta$. At the same time, the magnitude of

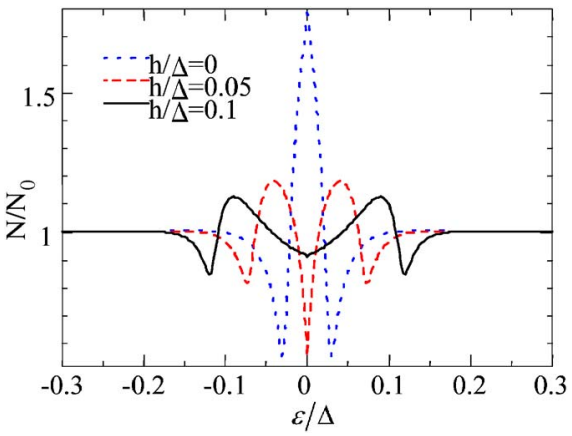

FIG. 7. (Color online) Normalized LDOS as a function of $\varepsilon$ for $R_{d} / R_{b}=0.1$ and various $h / \Delta$ in $p$-wave superconductor junctions.

the LDOS at $\varepsilon=0$ is most strongly suppressed (see Fig. 7).

Before ending this subsection, we investigate the crossover between singlet and triplet pairing states. Let us plot $f_{3}$ and $f_{0}$ for $\varepsilon=0$ as a function of $h$ at (a) $x=0$, (b) $x=L / 2$, and (c) $x=L$ as shown in Fig. 9. $f_{3}$ has a maximum at a certain value of $h$. When $h$ exceeds this value, the singlet component becomes dominant as shown in Fig. 9. The value of $h$ at the crossover increases with the increase of $Z, R_{d} / R_{b}$, and $E_{T h}$-i.e., with enhancement of the proximity effect.

\section{Relevance of the odd-frequency component to the ZEP of the LDOS}

Let us discuss the relation between the generation of the odd-frequency pairing and the ZEP in the LDOS, using general properties of solutions of the proximity effect problem. Since $\cos \bar{\theta}(\varepsilon)=\cos \theta^{*}(-\varepsilon)$ are satisfied, the LDOS normalized by its value in the normal state is given by

$$
N / N_{0}=\left[\cos \theta(\varepsilon)+\cos \theta^{*}(-\varepsilon)\right] / 2 .
$$

For $\varepsilon=0$, the normalized LDOS reads $\cosh [\operatorname{Im} \theta(0)] \cos [\operatorname{Re} \theta(0)]$, while $f_{3}(0)$ and $f_{0}(0)$ are given

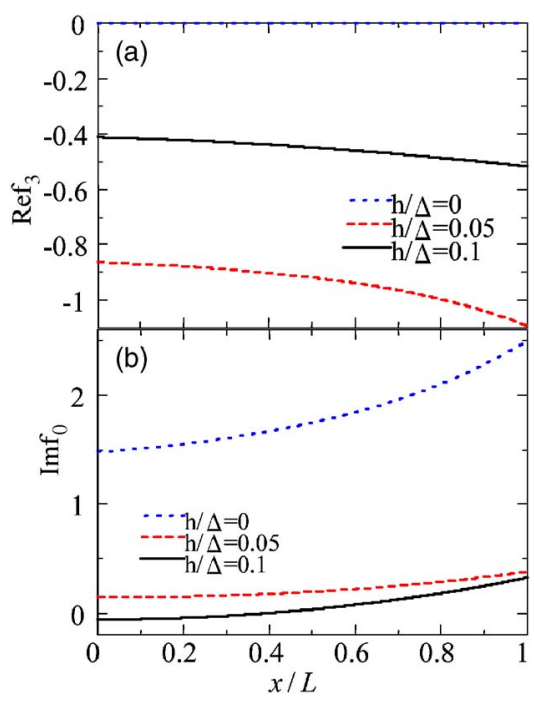

FIG. 8. (Color online) Spatial dependence of the pair amplitudes $f_{3}$ and $f_{0}$ in the DF for $\varepsilon=0$ in $p$-wave superconductor junctions. For $\varepsilon=0, \operatorname{Im} f_{3}=0$ and $\operatorname{Re} f_{0}=0$ are satisfied. 


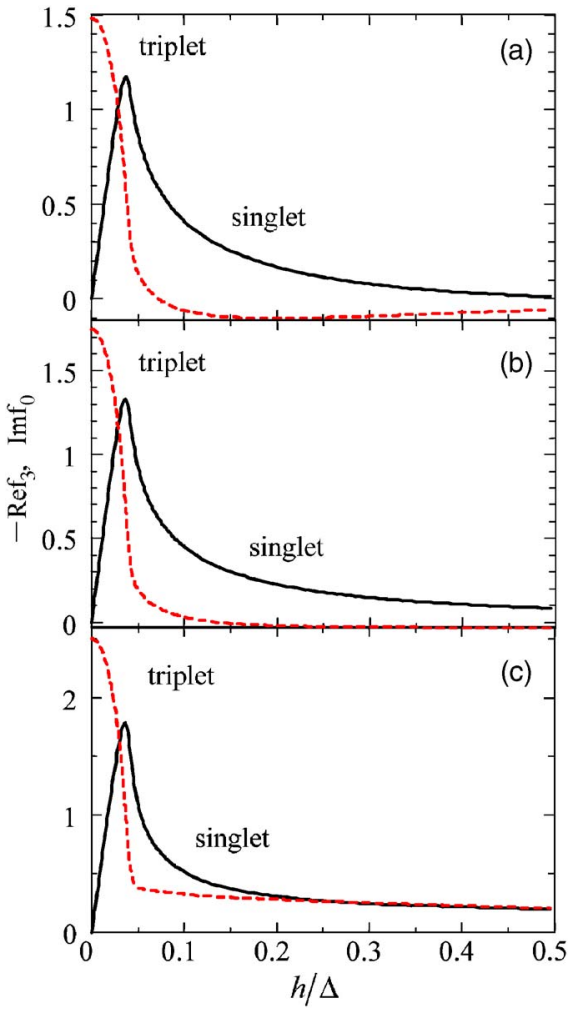

FIG. 9. (Color online) The pair amplitudes $f_{0}$ and $f_{3}$ as a function of $h$ in the DF for $\varepsilon=0$ in $p$-wave superconductor junctions. (a) $x=0$, (b) $x=L / 2$, and (c) $x=L$.

by $\quad \cosh [\operatorname{Im} \theta(0)] \sin [\operatorname{Re} \theta(0)] \quad$ and $\quad i \sinh [\operatorname{Im} \theta(0)]$ $\times \cos [\operatorname{Re} \theta(0)]$, respectively. As seen from these relations, $f_{0}$ becomes zero when the LDOS is zero. In addition, whether the spin-singlet component $f_{3}$ dominates the spin-triplet component $f_{0}$ or not crucially depends on the value of $\operatorname{Re} \theta(0)$. The most favorable condition where $N / N_{0}$ is enhanced is the large magnitude of $\operatorname{Im} \theta(0)$ and the absence of $\operatorname{Re} \theta(0)$, where $f_{0}$ dominates $f_{3}$. For the sufficiently large magnitude of $\operatorname{Im} \theta(0)$ and small magnitude of $\operatorname{Re} \theta(0)$, $N / N_{0} \sim \cos [\operatorname{Re} \theta(0)] \exp [\operatorname{Im} \theta(0)] / 2 \sim \exp [\operatorname{Im} \theta(0)] / 2$ and $f_{0}(0) \sim i \cos [\operatorname{Re} \theta(0)] \exp [\operatorname{Im} \theta(0)] / 2 \sim i \exp [\operatorname{Im} \theta(0)] / 2$ are satisfied. Then we obtain $N / N_{0} \sim-i f_{0}(0)$. This means that the generation of the odd-frequency pair amplitude $f_{0}(0)$ leads to the enhancement of the density of states at zero energy.

\section{CONCLUSIONS}

We have studied the proximity effect in diffusiveferromagnet/superconductor junctions. Various possible sym- metry classes in a superconductor were considered which are consistent with the Pauli's principle: the even-frequency spin-singlet even-parity state, even-frequency spin-triplet odd-parity state, odd-frequency spin-triplet even-parity state, and odd-frequency spin-singlet odd-parity state. As was established in the previous work, ${ }^{25}$ in the absence of the exchange field the induced pair amplitude in a DF belongs, respectively, to ESE, OTE, OTE, and ESE pairing states. It is shown in the present paper that, in addition to these states, the OTE, ESE, ESE, and OTE pairing states are generated in the DF in the presence of the exchange field $h$.

As a typical example of an ESE superconductor, we have chosen the spin-singlet $s$-wave state. We have clarified that when the OTE state dominates the ESE state in the DF, the resulting LDOS has a zero-energy peak. At the same time, the amplitude of the OTE pair wave function near the N/DF interface is enhanced at zero energy. As suggested by our findings, the odd-frequency pairing state was possibly realized in the experiment by Kontos et al.,,$^{5}$ where the ZEP was observed in ferromagnet/s-wave superconductor junctions.

We have also studied spin-triplet $p$-wave superconductor junctions. In this case, the ZEP in the LDOS splits into two peaks due to the generation of the ESE pairing state by the exchange field. The features of the proximity effect specific to spin-triplet $p$-wave superconductor junctions can be studied in experiments with the $\mathrm{Sr}_{2} \mathrm{RuO}_{4}-\mathrm{Sr}_{3} \mathrm{Ru}_{2} \mathrm{O}_{7}$ eutectic system. ${ }^{40}$ Based on general properties of solutions of the proximity effect problem, we have demonstrated that the generation of the odd-frequency pairing state at zero energy leads to the ZEP in the LDOS.

\section{ACKNOWLEDGMENTS}

The authors appreciate useful and fruitful discussions with M. Eschrig, Ya. V. Fominov, T. Akazaki, and H. Takayanagi. T.Y. acknowledges support by JSPS. This work was supported by a Grant-in-Aid for Scientific Research on Priority Area "Novel Quantum Phenomena Specific to Anisotropic Superconductivity" (Grant No. 17071007) from the Ministry of Education, Culture, Sports, Science and Technology of Japan. This work was also supported by NAREGI Nanoscience Project, the Ministry of Education, Culture, Sports, Science and Technology, Japan, the Core Research for Evolutional Science and Technology (CREST) of the Japan Science and Technology Corporation (JST), and a Grantin-Aid for the 21st Century COE "Frontiers of Computational Science." The computational aspect of this work has been performed at the Research Center for Computational Science, Okazaki National Research Institutes and the facilities of the Supercomputer Center, Institute for Solid State Physics, University of Tokyo.
${ }^{1}$ A. I. Buzdin, Rev. Mod. Phys. 77, 935 (2005).

${ }^{2}$ F. S. Bergeret, A. F. Volkov, and K. B. Efetov, Rev. Mod. Phys. 77, 1321 (2005).

${ }^{3}$ A. A. Golubov, M. Yu. Kupriyanov, and E. Il'ichev, Rev. Mod. Phys. 76, 411 (2004).
${ }^{4}$ V. V. Ryazanov, V. A. Oboznov, A. Yu. Rusanov, A. V. Veretennikov, A. A. Golubov, and J. Aarts, Phys. Rev. Lett. 86, 2427 (2001); V. V. Ryazanov, V. A. Oboznov, A. V. Veretennikov, and A. Yu. Rusanov, Phys. Rev. B 65, 020501(R) (2002); S. M. Frolov, D. J. Van Harlingen, V. V. Bolginov, V. A. Oboznov, and 
V. V. Ryazanov, ibid. 74, 020503(R) (2006); V. A. Oboznov, V. V. Bol'ginov, A. K. Feofanov, V. V. Ryazanov, and A. I. Buzdin, Phys. Rev. Lett. 96, 197003 (2006).

${ }^{5}$ T. Kontos, M. Aprili, J. Lesueur, and X. Grison, Phys. Rev. Lett. 86, 304 (2001); T. Kontos, M. Aprili, J. Lesueur, X. Grison, and L. Dumoulin, ibid. 93, 137001 (2004).

${ }^{6}$ H. Sellier, C. Baraduc, F. Lefloch, and R. Calemczuk, Phys. Rev. B 68, 054531 (2003).

${ }^{7}$ Y. Blum, A. Tsukernik, M. Karpovski, and A. Palevski, Phys. Rev. B 70, 214501 (2004).

${ }^{8}$ C. Surgers, T. Hoss, C. Schonenberger, and C. Strunk, J. Magn. Magn. Mater. 240, 598 (2002).

${ }^{9}$ C. Bell, R. Loloee, G. Burnell, and M. G. Blamire, Phys. Rev. B 71, 180501(R) (2005).

${ }^{10}$ V. Shelukhin, A. Tsukernik, M. Karpovski, Y. Blum, K. B. Efetov, A. F. Volkov, T. Champel, M. Eschrig, T. Lofwander, G. Schon, and A. Palevski, Phys. Rev. B 73, 174506 (2006).

${ }^{11}$ M. Weides, K. Tillmann, and H. Kohlstedt, Physica C 437-438, 349 (2006).

${ }^{12}$ H. Sellier, C. Baraduc, F. Lefloch, and R. Calemczuk, Phys. Rev. Lett. 92, 257005 (2004).

${ }^{13}$ F. S. Bergeret, A. F. Volkov, and K. B. Efetov, Phys. Rev. Lett. 86, 4096 (2001).

${ }^{14}$ A. F. Volkov, A. Anishchanka, and K. B. Efetov, Phys. Rev. B 73, 104412 (2006); A. F. Volkov, Ya. V. Fominov, and K. B. Efetov, ibid. 72, 184504 (2005); F. S. Bergeret, A. F. Volkov, and K. B. Efetov, ibid. 68, 064513 (2003).

${ }^{15}$ A. Kadigrobov, R. I. Shekter, and M. Jonson, Europhys. Lett. 90, 394 (2001).

${ }^{16}$ M. Eschrig, J. Kopu, J. C. Cuevas, and G. Schön, Phys. Rev. Lett. 90, 137003 (2003); T. Löfwander, T. Champel, J. Durst, and M. Eschrig, ibid. 95, 187003 (2005).

${ }^{17}$ Y. V. Fominov, A. A. Golubov, and M. Y. Kupriyanov, JETP Lett. 77, 510 (2003).

${ }^{18}$ M. L. Kulic and M. Endres, Phys. Rev. B 62, 11846 (2000).

${ }^{19}$ R. S. Keizer, S. T. B. Goennenwein, T. M. Klapwijk, G. Miao, G. Xiao, and A. Gupta, Nature (London) 439, 825 (2006).

${ }^{20}$ I. Sosnin, H. Cho, V. T. Petrashov, and A. F. Volkov, Phys. Rev. Lett. 96, 157002 (2006).
${ }^{21}$ V. L. Berezinskii, JETP Lett. 20, 287 (1974).

${ }^{22}$ A. Balatsky and E. Abrahams, Phys. Rev. B 45, 13125 (1992); E. Abrahams, A. Balatsky, D. J. Scalapino, and J. R. Schrieffer, ibid. 52, 1271 (1995).

${ }^{23}$ M. Vojta and E. Dagotto, Phys. Rev. B 59, R713 (1999).

${ }^{24}$ Y. Fuseya, H. Kohno, and K. Miyake, J. Phys. Soc. Jpn. 72, 2914 (2003).

${ }^{25}$ Y. Tanaka and A. A. Golubov, Phys. Rev. Lett. 98, 037003 (2007).

${ }^{26}$ C. R. Hu, Phys. Rev. Lett. 72, 1526 (1994); Y. Tanaka and S. Kashiwaya, ibid. 74, 3451 (1995); S. Kashiwaya and Y. Tanaka, Rep. Prog. Phys. 63, 1641 (2000) and references therein.

${ }^{27}$ Y. Tanaka and S. Kashiwaya, Phys. Rev. B 70, 012507 (2004); Y. Tanaka, S. Kashiwaya, and T. Yokoyama, ibid. 71, 094513 (2005); Y. Tanaka, Y. Asano, A. A. Golubov, and S. Kashiwaya, ibid. 72, 140503(R) (2005); Y. Asano, Y. Tanaka, and S. Kashiwaya, Phys. Rev. Lett. 96, 097007 (2006).

${ }^{28}$ A. A. Golubov, M. Yu. Kupriyanov, and Ya. V. Fominov, JETP Lett. 75, 223 (2002).

${ }^{29}$ A. Buzdin, Phys. Rev. B 62, 11377 (2000).

${ }^{30}$ M. Zareyan, W. Belzig, and Yu. V. Nazarov, Phys. Rev. Lett. 86, 308 (2001); Phys. Rev. B 65, 184505 (2002).

${ }^{31}$ I. Baladie and A. Buzdin, Phys. Rev. B 64, 224514 (2001).

${ }^{32}$ F. S. Bergeret, A. F. Volkov, and K. B. Efetov, Phys. Rev. B 65, 134505 (2002).

${ }^{33}$ T. Yokoyama, Y. Tanaka, and A. A. Golubov, Phys. Rev. B 72, 052512 (2005); 73, 094501 (2006).

${ }^{34}$ J. W. Serene and D. Rainer, Phys. Rep. 101, 221 (1983).

${ }^{35}$ M. Eschrig, Phys. Rev. B 61, 9061 (2000).

${ }^{36}$ K. D. Usadel, Phys. Rev. Lett. 25, 507 (1970).

${ }^{37}$ Y. Tanaka, A. A. Golubov, and S. Kashiwaya, Phys. Rev. B 68, 054513 (2003).

${ }^{38}$ A. F. Volkov, A. V. Zaitsev, and T. M. Klapwijk, Physica C 210, 21 (1993).

${ }^{39}$ S. Yip, Phys. Rev. B 52, 3087 (1995).

${ }^{40}$ J. Hooper, M. Zhou, Z. Q. Mao, Y. Liu, R. Perry, and Y. Maeno, Phys. Rev. B 73, 132510 (2006); R. Fittipaldi, A. Vecchione, S. Fusanobori, K. Takizawa, H. Yaguchi, J. Hooper, R. S. Perry, and Y. Maeno, J. Cryst. Growth 282, 152 (2005). 